\title{
Content-Based Image Retrieval Using Shifted Histogram
}

\author{
Gi-Hyoung Yoo ${ }^{1}$, Beob Kyun $\mathrm{Kim}^{2}$, and Kang Soo You ${ }^{3}$ \\ ${ }^{1}$ Department of Computer Engineering, Chonbuk National University, \\ Jeonju, 561-756, Korea \\ ghryoo@gmail.com \\ ${ }^{2}$ Korea Institute of science and technology Information, Daejeon, 350-806, korea \\ Bkyun.kimagmail.com \\ ${ }^{3}$ School of Liberal Arts, Jeonju University, Jeonju, 561-756, Korea \\ kangsoo.you@gmail.com
}

\begin{abstract}
This paper proposes the shifted histogram method (SHM), for histogram-based image retrieval based on the dominant colors in images. The histogram-based method is very suitable for color image retrieval because retrievals are unaffected by geometrical changes in images, such as translation and rotation. Images with the same visual information, but with shifted color intensity, may significantly degrade if the conventional histogram intersection method (HIM) is used. In order to solve this problem, we propose the shifted histogram method (SHM). Our experimental results show that the shifted histogram method
\end{abstract}

Keywords: Content-Based Image Retrieval, Color Histogram, Shift Histogram.

\section{Introduction}

Content-based image retrieval (CBIR) is a promising approach to search through an image database by means of image feature, such as appearance, color, texture, shape, pattern, or a combination these factors [1], [2], [3], [4]. In the Histogram intersection method (HIM) [5], [6] is the most common approach for using the intersected area of histograms acquired from image features.

Although the conventional color histogram method can describe the global color distribution of images in a straightforward manner, it only provides a very coarse characterization of images. There is no spatial information included in the color histogram. Thus, two completely different images may have very similar histograms. Also, the lighting conditions will alter the histogram of an image. Even two pictures with little difference in lighting conditions can not easily be matched.

In PWH (perceptually weighted histogram) by Lu et al. [7], each color from an image is represented by its 10 nearest similar colors. However, HIM still takes place of the full color-range (e.g. 256 colors) of the two images being compared. In MHM (merged histogram method), colors from individual images and between images are merged to form a dominant color set, instead of color components. The idea behind MHM is to retrieve images in a database of which the intensity has been changed by 
light. However, this method requires greater processing capability to generate feature vectors.

In this paper, we propose a novel shifted histogram method (SHM), to overcome the disadvantages of the conventional histogram method.

\section{Histogram-Based Color Image Retrieval}

The histogram-based method is very suitable for color image retrieval, because this method is unaffected by geometrical information in images, such as translation and rotation. The histogram intersection method (HIM) [5],[6] measures the intersections between the two image histograms. These intersections are usually named query image for the query input and target image for the image database. An image histogram is an $n$-dimensional vector, in which each element represents the number of pixels of color in the n-color image.

Regardless of image size, each element is normalized before comparison. The similarity measure is then calculated by calculating the histogram intersection, as shown in equation (1).

$$
I(T, Q)=\sum_{k=1}^{n} \min \left(T_{k}, Q_{k}\right)
$$

The larger the value has, the more similar the image has in equation (1). Images can then be ranked from the image database.

\section{Shifted Histogram Method (SHM)}

In a more general mathematical sense, a histogram is simply a mapping that counts the number of observations that fall into various disjoint categories (known as bins), whereas the graph of a histogram is merely one way to represent a histogram. Thus, if we let the total number of observations be the total number of bins, the histogram meets the following conditions in figure 1 .

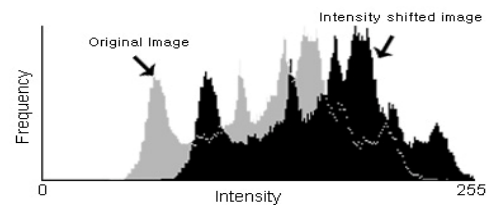

Fig. 1. Histogram of original image and shifted image by light change

The proposed SHM can be divided into 4 stages before similarity measurement. The process is as follows: 


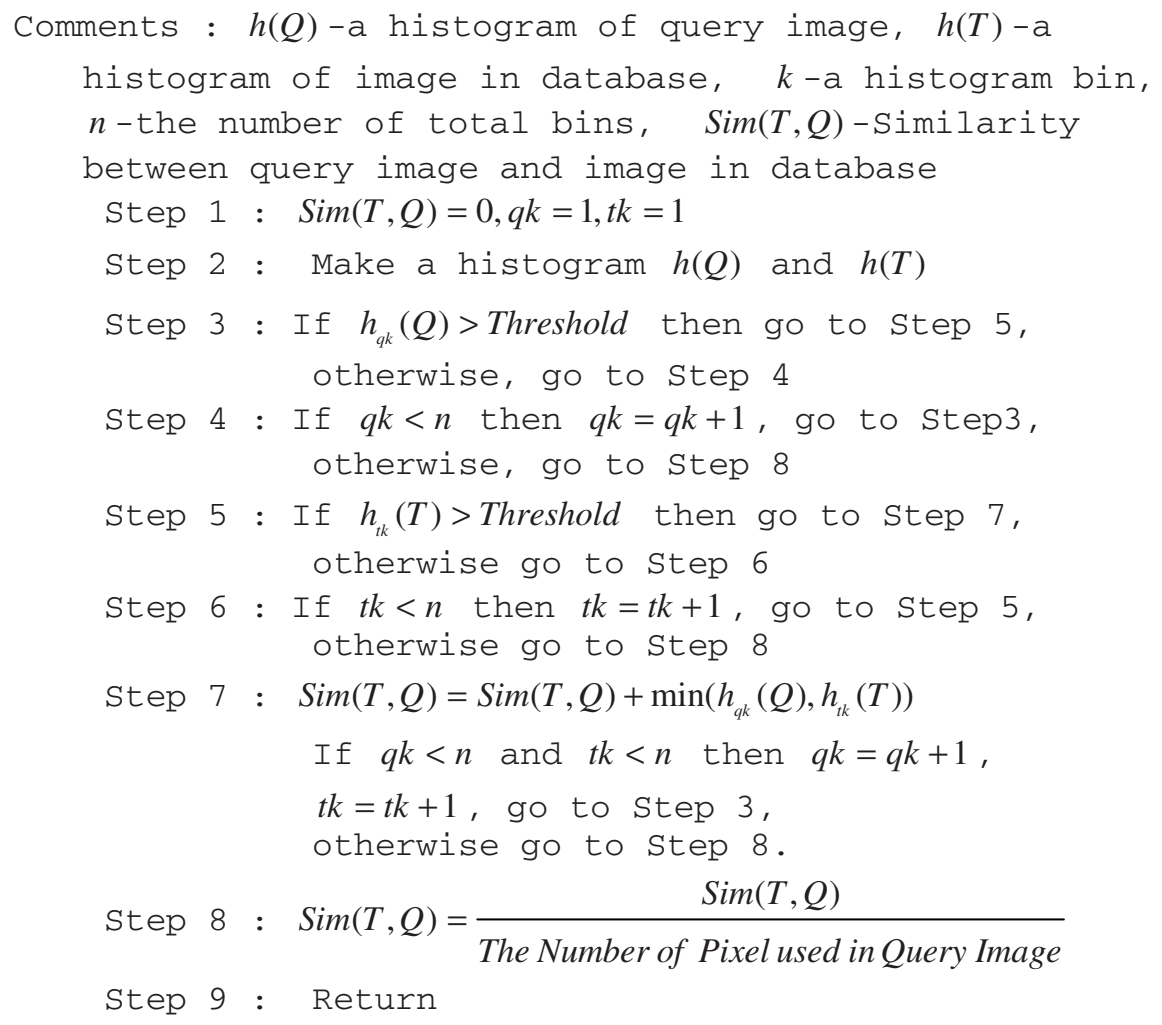

\section{Experiments}

The analysis of histogram effectiveness in image discrimination is mainly used for the design of image retrieval systems. In order to test the performance of our method, we have implemented several groups of experiments. The testing image database includes images with a wide range of image content, such as nature scenes, flowers, animals, cars, trains, and so on.

The performance of the retrieval results is measured by Precision, Recall [8] and AVRR (AVerage Rank of Relevant images). Relevant images are referred to as images in the same class. In table 1 and table 2, the performance of SHM, MCH and CHM is compared in terms of precision, recall and AVRR.

Table 1. Result of the three methods in flowers

\begin{tabular}{lllll}
\hline Method & Result & Recall & Precision & AVRR \\
\hline SHM & 7 & 0.78 & 0.67 & 4.2 \\
MCH & 6 & 0.59 & 0.61 & 4.0 \\
CHM & 4 & 0.47 & 0.51 & 3.5
\end{tabular}


Table 2. Result of the three methods in trains

\begin{tabular}{lllll}
\hline Method & Result & Recall & Precision & AVRR \\
\hline SHM & 7 & 0.78 & 0.67 & 4.2 \\
MCH & 6 & 0.59 & 0.61 & 4.0 \\
CHM & 4 & 0.47 & 0.51 & 3.5
\end{tabular}

\section{Conclusion}

The shifted-color histogram method (SHM) is proposed for histogram-based image retrieval, and is based on the dominant colors in images. The histogram-based method is very suitable for color image retrieval because it is unaffected by geometrical changes in images, such as translation and rotation. However, images with similar visual information but with shifted color intensity, may result in a significant degradation in the similarity level, if the conventional histogram intersection method (HIM) is used. To solve the problem, the shifted histogram method (SHM) is proposed.

In comparison with other methods, our method is more straightforward. It doesn't haves special requirements or extra restrictions for images, for content-based image retrieval. Our experimental results show that the shifted histogram method has significantly higher retrieval effectiveness than the standard histogram method. In future study, the proposed retrieval method is expected to be applied to large scale image and video databases.

\section{References}

1. Flickner, M. et al:: Query by image and video content: The QBIC system. IEEE Computer, Vol. 28(9). (1995) 23-32

2. Swain, M.J., Ballard, D.H.: Color indexing. Int. J. Computer Vision, Vol. 7(1). (1991) $11-32$.

3. Gagaudakis, G., Rosin, P.: Incoprerating shape into histograms for CBIR. Proc. Int. Conf. Image Processing, Thessaloniki, Greece, (2001) 7-10

4. Rui, Y., Huang, T., Chang, S.F.: Image Retrieval:Current Techniques, Promising Directions and Open Issues. J. Visual Communication and Image Representation, Vol. 10. (1999) 39-62

5. Wong, K.M., Cheung, C.H., Po, L.M.: Dominant Color Image Retrieval using Merged Histogram. Proc. the 2003 Int. Symposium, (2003)

6. Wong, K.M., Cheung, C.H., Po, L.M.: Merged-color histogram for color image retrieval. Proc. of the 2002 Int. Conference, (2002) 24-28

7. Seong, O.S., Choi, T.S.: Edge Color Histogram for Image Retrieval. IEEE ICIP 0-78037622-6/02/ (2000)

8. Muller, H., Muller, W., Squire D., Marchand, M.S., Pun, T.: Performance Evaluation in Content-Based Image Retrieval:Overview and Proposals. Pattern Recognition Letters, Vol. 22(5). (2001) 593-601 